Check for updates

Cite this: RSC Adv., 2017, 7, 53265

Received 28th August 2017

Accepted 13th November 2017

DOI: 10.1039/c7ra09551a

rsc.li/rsc-advances

\section{Sensing mechanism of an ionization gas temperature sensor based on a carbon nanotube film}

\author{
Hui Song, (D) *ab Yong Zhang ${ }^{b}$ and Jiaxing Cao ${ }^{b}$
}

In this paper, we propose a novel ionized gas temperature sensor which has a triple-electrode structure and carbon nanotubes (CNTs) as a field emission cathode. The measurement current, as part of the non-selfsustaining discharge current, changes with the changing of initial emission current $I_{0}$ and Townsend's first ionization coefficient $\alpha$ according to the theory of Townsend discharge. The ionization coefficient $\alpha$ increases with increasing gas temperature for a given gas at a certain electric field because the mean free path is proportional to the gas temperature. In addition, the initial current increases with increasing gas temperature. Experimental results showed that the collecting current exhibited an exponential increase with the gas temperature rising from $20{ }^{\circ} \mathrm{C}$ to $110{ }^{\circ} \mathrm{C}$ and the sensitivity is $4.74 \mu \mathrm{A}{ }^{\circ} \mathrm{C}^{-1}$ to air and $22.72 \mu \mathrm{A}{ }^{\circ} \mathrm{C}^{-1}$ to nitrogen at $110^{\circ} \mathrm{C}$. The difference in the effective work function after nitrogen and oxygen adsorption is the response for the different sensitivities. The triple-electrode ionization sensor is a new mechanism of gas temperature measurement and has the merit of facilitating the fabrication of CNTs compared with the existing CNT-based resistive or thermal expansion temperature sensors.

\section{Introduction}

Carbon nanotubes (CNTs) have been shown to have various potential applications ${ }^{1-6}$ and have attracted significant interest due to their very attractive mechanical and electrical properties. Recent studies have shown that carbon nanotubes (CNTs) can be used as temperature sensors ${ }^{7-12}$ because the extremely small size of the CNT can provide accurate measurements at nanoscale size and reduce the possibility of disturbing the neighboring environment, which is very important in a complicated thermal flow system. The reported CNT-based temperature sensors can be categorized into thermal expansion and thermal resistance according to their different working principles.

The thermal expansion CNT-based temperature sensor was firstly proposed by investigating the thermal expansion behavior of loading metal gallium into the CNTs. ${ }^{13}$ Due to their low melting point, metal indium ${ }^{14}$ and $\mathrm{Ge}^{15}$ were also loaded into CNTs as the thermal indicator for temperature measurement. Although the thermal expansion coefficient of the filling metal varies linearly and reproducibly with values corresponding to macroscopic volumes in range of temperature measurement, measurements for a single carbon nanotube are extremely difficult, which makes their use very complicated. $^{10,11,16}$ The thermal resistance CNT-based temperature

${ }^{a}$ College of Engineering, Qufu Normal University, Rizhao, Shandong, 276826, China. E-mail: songh_0315@mail.qfnu.edu.cn

${ }^{b}$ State Key Laboratory of Electrical Insulation and Power Equipment, Xi'an Jiaotong University, Xi'an, Shaanxi, 710049, China sensors were based on the conductivity change of CNTs induced by the thermal exchange., ${ }^{9,12,17-23}$ A long single-walled CNT (SWCNT) array suspended between two separated metal electrodes was used for temperature measurement and the resistance of the SWCNT array is proportional to the temperature. ${ }^{22}$ Due to the difficulty of placing individual CNTs at desired locations and the integration of nanostructures, this CNT-based temperature sensor has been deemed impractical for commercialization. ${ }^{24}$ Y. Hong et al. connected two parallel silver electrodes via PDMS-encapsulated CNT thin film to fabricate a temperature sensor and measured the change in CNT-film resistance according to temperature variation. ${ }^{25}$ And A. Di Bartolomeo et al. presented the electric resistance of the thick and dense carbon nanotube networks in form of freestanding films (CNTFs) as a function of the temperature from 4 to $420 \mathrm{~K}^{12}$ However, the applied electric field and the area of the CNTs have considerable effect on the sensitivity of those gas temperature sensors.

Since 2008, a novel CNT film-based ionization sensor has been proposed for gas detection in our lab. ${ }^{26-30}$ The tripleelectrode configuration enables a long CNTs life, which ensures the ionization sensor operating in non-self-sustaining discharge state for a long time. The measurement current, as part of non-self-sustaining discharge current, varies with the initial emission current $I_{0}$ and the degree of ionization $\alpha$. Due to the effect of gas temperature on the ionization coefficient $\alpha$ and the emission $I_{0}$, the collecting current should be varied with gas temperature. This is a new mechanism of gas temperature measurement comparing with all previously reported CNT- 
based temperature sensor. Here, the novel CNT film-based ionization sensor is used for the monitoring of flue gas temperature in the power plant, which is generally within $110{ }^{\circ} \mathrm{C}$.

\section{Experimental details}

\subsection{Fabrication of gas temperature sensor}

A triple-electrode gas temperature sensor is designed and fabricated shown in Fig. 1(a). Three silicon slices in size of $27 \mathrm{~mm} \times 8 \mathrm{~mm} \times 450 \mu \mathrm{m}$ are processed through mask, photolithography, dry etching and cleaning for the preparation of electrodes. There are two cooling holes of $4 \mathrm{~mm}$ diameter in cathode, one $3 \mathrm{~mm}$ radial round hole for ions migration and diffusion in extracting electrode and a square blind rectangular of $8 \times 6 \mathrm{~mm}^{2}$ in area and $200 \mu \mathrm{m}$ in depth for collecting positive ions in collecting electrode, respectively (Fig. 1(b)). Ti/Ni/Au (50 nm/125 nm/400 nm) are sputtered in sequence on both sides of extracting electrode, the inner side of cathode and collecting electrode. Then three electrodes are rapidly annealed at $450{ }^{\circ} \mathrm{C}$ for about 50 seconds to enhance the bond strength of the substrate and the $\mathrm{Ti} / \mathrm{Ni} / \mathrm{Au}$ film. And next vertically aligned carbon nanotube array is grown on $\mathrm{SiO}_{2} / \mathrm{Si}$ substrate by thermal chemical vapor deposition (TCVD) method and transferred to the inner side of cathode by wetting transfer method. ${ }^{31}$ Three electrodes are separated with $170 \mu \mathrm{m}$-thick polyester film and connected with three golden wires as three pins of the sensor. The images of the CNT film is scanned with the transmission electron microscopy (FEI Quanta 250 FEG) and here we give the image of the CNTs film as shown in Fig. 1(c).

\subsection{Experimental system}

Gas temperature sensing experiment is conducted in a test chamber based on the NI PXI-1044 test system. The stable voltages applying between cathode, the extracting electrode and

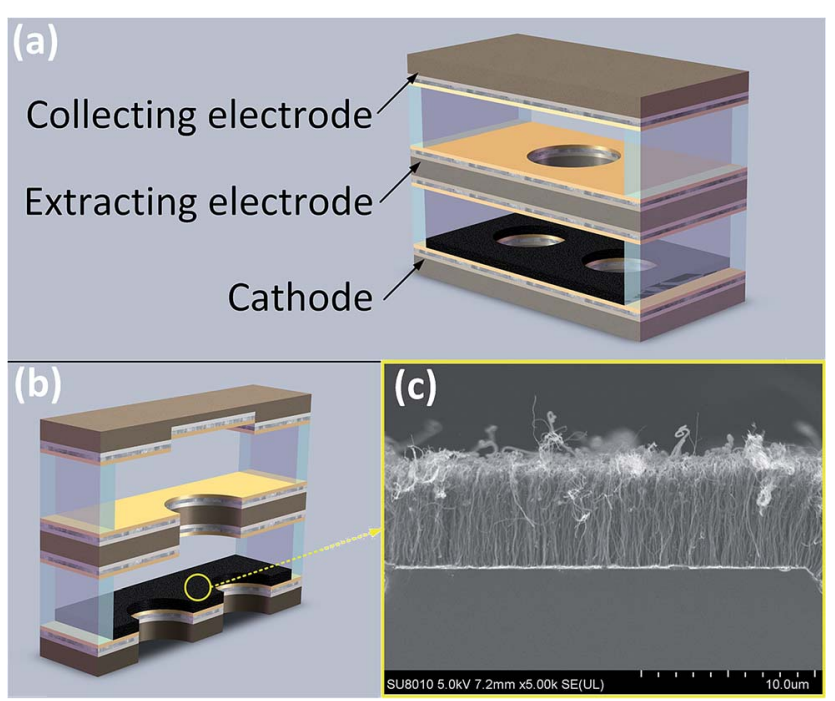

Fig. 1 (a) Scheme of the triple-electrode CNT-based sensor (b) sectional view of the sensor (c) FE-SEM image of CNT film. the collecting electrode are supplied by power modules (NI PXI4132). The collecting currents $I_{\mathrm{c}}$ are recorded by a precisely digital multimeters (NI PXI-4071) controlled by computer via the MXI interface of test system. The fabricated sensor is placed into the test chamber which could be heated by a heating resistor. When temperature of the chamber varied from $20^{\circ} \mathrm{C}$ to $110^{\circ} \mathrm{C}$, the collecting current was recorded by the computer via the MXI interface of test system as shown in Fig. 2.

\section{Experimental results and discussion}

\subsection{Gas temperature sensing}

The $I_{\mathrm{c}}-T$ characteristic of the ionization gas temperature sensor was tested in $\mathrm{N}_{2}$ at a fixed $U_{1}$ of $10 \mathrm{~V}$. When the gas temperature $T$ rises from $25{ }^{\circ} \mathrm{C}$ to $70{ }^{\circ} \mathrm{C}$, the sensor shows an exponential increasing responses to temperature. The collecting currents $I_{\mathrm{c}}$ increase with the extracting voltage $U_{2}$ increasing from $30 \mathrm{~V}$ to $70 \mathrm{~V}$ at each gas temperature as well. All $I_{\mathrm{c}}-T$ characteristic curves have nearly the same shape (black, red, blue and rose red curves in Fig. 3(a)). This indicates that the novel ionization temperature sensor is capable of gas temperature measurement and has excellent reproducibility. In addition, the higher the extracting voltage $U_{2}$, the better gas temperature response. Considering the triple-electrode sensor working under the nonself-sustaining discharge state, the applied extracting voltage $U_{2}$ should be lower than breakdown voltage. While the sensor needs a higher voltage to gain better temperature response and a lower one to reduce the power consumption. So it is a tradeoff between better response and lower power consumption.

The same temperature responses shape at different extracting voltages $U_{2}$ (Fig. 3(a)) has demonstrated the good stability of the sensor. To verify the long-term stability, the temperature sensing experiment of the sensor was repeated at least one month later. The almost negligible difference between the two gas temperature responses further confirms the stability of the sensor (Fig. 3(b))

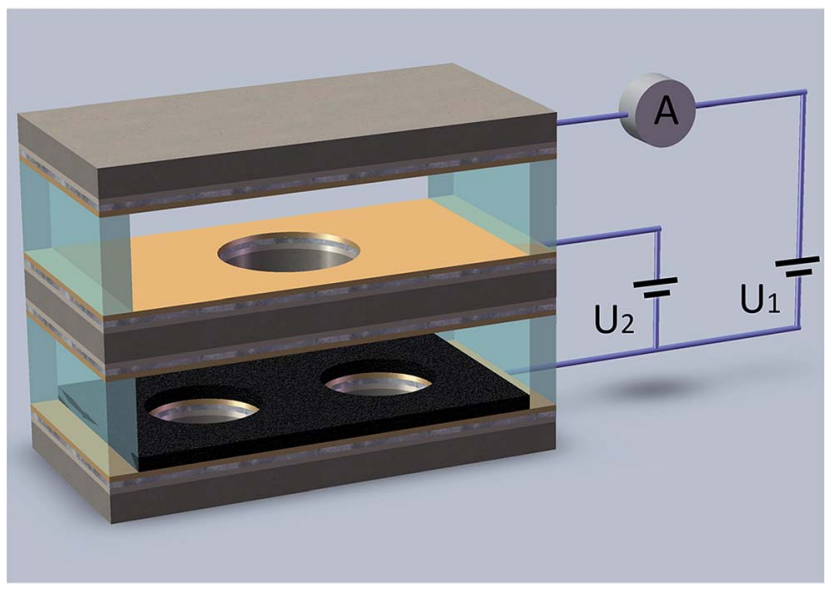

Fig. 2 Schematic diagram of the test system $\left(U_{1}, U_{2}\right.$ is the voltage applied between the cathode and the collecting electrode and between the cathode and the extracting electrode, respectively). 

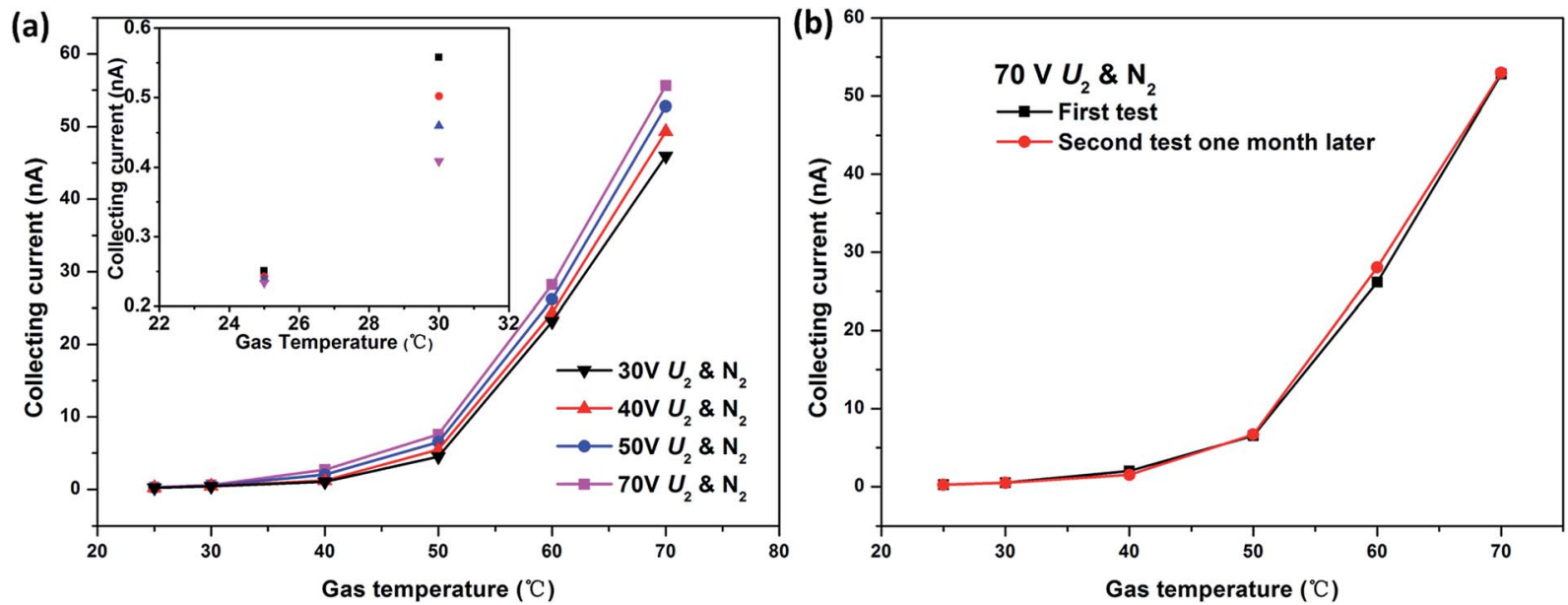

Fig. 3 (a) Effect of extracting voltage $U_{2}$ on gas temperature sensing properties and (b) long-term stability of novel gas temperature sensor in nitrogen

Fixed $U_{1}$ at $10 \mathrm{~V}$ and $U_{2}$ at $70 \mathrm{~V}$, the gas temperature experiments were conducted in air and nitrogen, respectively. When the gas temperature rises from $20{ }^{\circ} \mathrm{C}$ to $110{ }^{\circ} \mathrm{C}$, the collecting current exhibits an exponential increase as shown in Fig. 4(a) and (b). According to the definition of sensitivity $(S=\Delta y / \Delta x$, where $\Delta y$ is the variation of the collecting current and $\Delta x$ is the variation of gas temperature), the sensitivity of the tripleelectrode ionization sensor is $4.74 \mu \mathrm{A}{ }^{\circ} \mathrm{C}^{-1}$ to air and $22.72 \mu \mathrm{A}{ }^{\circ} \mathrm{C}^{-1}$ to nitrogen at $110{ }^{\circ} \mathrm{C}$, respectively. It is obvious that the novel ionization gas temperature sensor is more sensitive to $\mathrm{N}_{2}$ than air (Fig. 4(a) and (b)).

According to the theory of Townsend discharge, as part of discharge current, the collecting current $I_{\mathrm{c}}$ can be expressed as follow

$$
I_{\mathrm{c}}=I_{0} e^{\alpha d}
$$

where $I_{0}$ is initial emission current of CNTs cathode, $d$ electrode separation between cathode and the extracting electrode and $\alpha$ Townsend's first ionization coefficient. Eqn (1) showed that the initial emission current $I_{0}$ and Townsend's first ionization coefficient $\alpha$ have effect on the collecting current at constant electrode separation $d$.

\subsection{Effect of initial emission current $I_{0}$}

When a stable DC voltage applied between the cathode and the extracting electrode, a non-uniform field is generated between them. It is significantly enhanced near the nanotube tips and become much higher than the other region. This enable initial electrons to be continuously emitted from the CNTs cathode. Under the joint action of the electric field and temperature, the electrons are accelerated during the movement to anode, collide with gas molecules, and consequently free additional electrons. Those electrons are in turn accelerated and collide with gas molecules. Accordingly, a large number of ions generate in the vicinity of CNTs cathode due to the collision ionization between high speed electrons and gas particles. Because of the different ions concentration, part of positive ion can be extracted from the cathode region through the extracting hole toward the collecting electrode and be measured as collecting current.
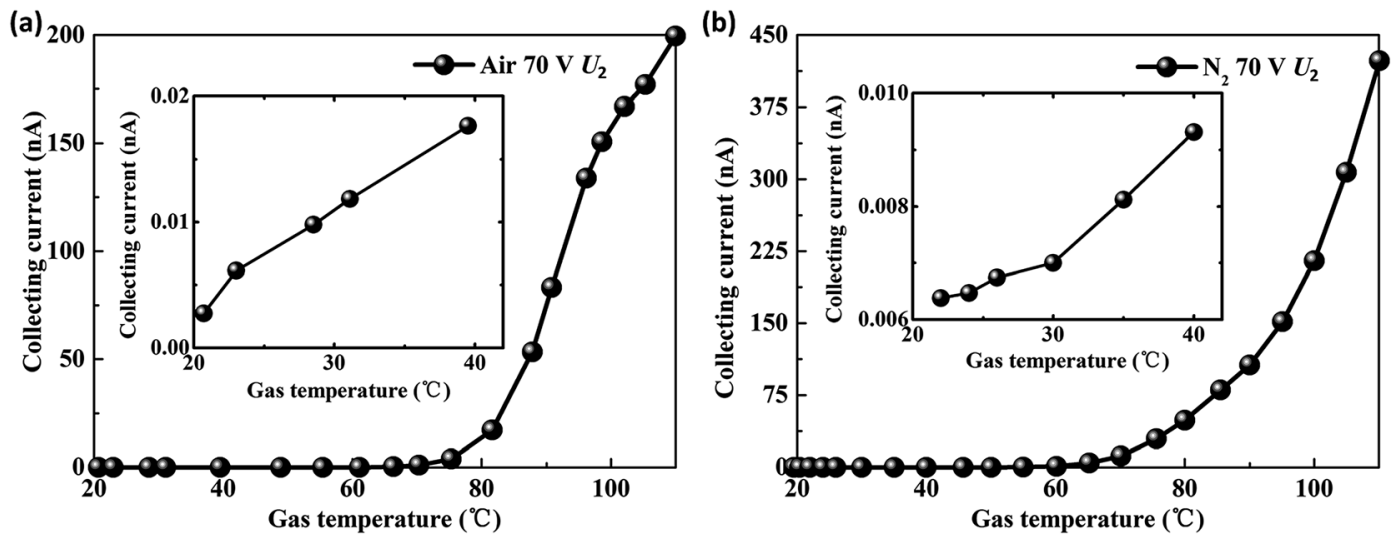

Fig. 4 Collecting current changes with gas temperature in (a) air and (b) nitrogen. 
Y. Zhang et al. ${ }^{29}$ have proved that the emission of the tripleelectrode sensor is field-assisted thermal emission and the initial emission current density could be expressed as follow:

$$
j_{\text {Schottky }}=A T^{2} \exp \left(\frac{-\varphi_{\text {eff }}}{k_{\mathrm{B}} T}\right)
$$

where $A=1.20173 \times 10^{6} \mathrm{~A} \mathrm{~m}^{-2} \mathrm{~K}^{-2}$ is Richardson constant, $k_{\mathrm{B}}=1.38065 \times 10^{-23} \mathrm{~J} \mathrm{~K}^{-2}$ is Boltzmann constant and $\varphi_{\text {eff }}$ is the effective work function of CNTs after gas adsorption.

The initial emission current $I_{0}=j_{\text {schottky }} \times \mathrm{SN}$ exponentially increases with increasing emission current density $j_{\text {schottky }}$ (where SN is the effective emission area and constant here). On the one hand, the initial current density $j_{\text {schottky }}$ increases with increasing temperature when the effective work functions of CNTs is determined after certain gas adsorption. On the other hand, the effective work function $\varphi_{\text {eff }}$ also has effect on the initial current density considering the different effective work function of CNTs after different gas adsorption. ${ }^{32-35}$

The work function of pristine CNTs is reported to varied from 4.8 to $5.1 \mathrm{eV}$ (ref. 36-40) and rises to the average value of $6.22 \mathrm{eV}$ after oxygen adsorption, but does not goes up significantly after nitrogen adsorption. ${ }^{33,38}$ While the greater the effective work function, the smaller the current density according to eqn (2). That is why the ionization sensor is more sensitive to $\mathrm{N}_{2}$ than air because that the air exposure effect should be dominated by oxygen. ${ }^{35}$

\subsection{Effect of Townsend's first ionization coefficient $\alpha$}

Townsend ionization is a gas ionization process where free electrons are accelerated by an electric field, collide with gas molecules. Only when the energy picked up by an electron in its mean free path $\lambda$ along field $E$ is greater than the ionization potential $\varphi_{\mathrm{i}}$ of gas molecule, the impact ionization occurs and the probability of ionization is proportion to $\exp \left(-\varphi_{\mathrm{i}} / E \lambda\right)$. The probability of ionization, that is, ionization coefficient $\alpha$, is characterized by the number of ionization events performed by an electron in its mean free path $\lambda$ along electric field $E$.

$$
\alpha \propto \exp \left(-\varphi_{\mathrm{i}} / E \lambda\right)
$$

The probability of ionization $\alpha$ varies with the mean free path $\lambda$ of electron and gas molecule collision at a constant electric field $E$ for a given gas due to its constant ionization potential $\varphi_{\mathrm{i}}$ according to eqn (3). The mean free path $\lambda$ increases with increasing gas temperature $T$ according to kinetic theory expression $\lambda=k_{\mathrm{B}} T / p \sigma$ with Boltzmann constant $k_{\mathrm{B}}$, constant gas pressure $p$ and effective cross-sectional area for collision $\sigma$. Therefore the ionization coefficient $\alpha$ increases with increasing gas temperature due to the increasing mean free path according to eqn (3), resulting in an exponential increasing in collecting current $I_{\mathrm{c}}$ according to eqn (1). The collecting current of our experimental results are in good agreement with the theoretical analysis. This is a new insight of gas temperature measurement.

\section{Conclusion}

A triple-electrode CNT-based ionization sensor was fabricated for gas temperature measurement. By applying suitable voltages $U_{2}$ and $U_{1}$, the novel ionization sensor could work in non-selfsustaining gas discharge state for a long time. The collecting current, as part of discharge current, changes with gas temperature because that the initial current and the first ionization coefficient are functions of gas temperature according to Townsend discharge. Experiment result shows that the collecting current exhibited an exponential increase with the gas temperature. The sensor can accurately detect gas temperature in range of $20-110^{\circ} \mathrm{C}$ with highest sensitivity of $4.74 \mu \mathrm{A}^{\circ} \mathrm{C}^{-1}$ to air and $22.72 \mu \mathrm{A}^{\circ} \mathrm{C}^{-1}$ to nitrogen at $110^{\circ} \mathrm{C}$, respectively. The gas temperature also shows excellent reproducibility and long-term stability. Compared with the existing CNT-based resistive or thermal expansion temperature sensors, ionization gas temperature sensors facilitate the fabrication of CNTs.

\section{Conflicts of interest}

The authors declare that there is no conflict of interests regarding the publication of this study.

\section{Acknowledgements}

This study was financially supported by grants from the National Science Foundation of China (50877056). Metal sputtering and CNTs growth were done in Institute of Vacuum Microelectronics \& microelectromechanical System, Xi'an Jiaotong University, Xi'an, China; Authors also thank Prof. Li for her help in CNTs growth.

\section{References}

1 A. Cismaru, M. Aldrigo, A. Radoi and M. Dragoman, Carbon nanotube-based electromagnetic band gap resonator for CH4 gas detection, J. Appl. Phys., 2016, 119, 124504.

2 S.-J. Young and Z.-D. Lin, Sensing Performance of Carbon Dioxide Gas Sensors with Carbon Nanotubes on Plastic Substrate, ECS J. Solid State Sci. Technol., 2017, 6, M72-M74.

3 L. Yunhan, S. Yonghai, D. A. Jaffray and J. T. W. Yeow, A novel field emission microscopy method to study field emission characteristics of freestanding carbon nanotube arrays, Nanotechnology, 2017, 28, 155704.

4 D. Zhang, K. Wang, J. Tong and B. Xia, Characterization of layer-by-layer nano self-assembled carbon nanotube/ polymer film sensor for ethanol gas sensing properties, Microsyst. Technol., 2014, 20, 379-385.

5 D. Zhang, Y. Sun and Y. Zhang, Fabrication and characterization of layer-by-layer nano self-assembled $\mathrm{ZnO}$ nanorods/carbon nanotube film sensor for ethanol gas sensing application at room temperature, J. Mater. Sci.: Mater. Electron., 2015, 26, 7445-7451.

6 D. Zhang, K. Wang, J. Tong and B. Xia, Layer-by-Layer Nanoassembly Fabrication and Humidity Sensing Behaviors of Multi-Walled Carbon Nanotubes/ 
Polyelectrolyte Hybrid Film, J. Nanosci. Nanotechnol., 2016, 16, 6705-6710.

7 A. D. Bartolomeo, et al., Multiwalled carbon nanotube films as temperature nano-sensors, NSTI-Nanotech., 2008, 1, 4.

8 A. Saraiya, D. Porwal, A. N. Bajpai, N. K. Tripathi and K. Ram, Investigation of Carbon Nanotubes as Low Temperature Sensors, Synth. React. Inorg., Met.-Org., Nano-Met. Chem., 2006, 36, 163-164.

9 M. Sibinski, M. Jakubowska and M. Sloma, Flexible temperature sensors on fibers, Sensors, 2010, 10, 7934.

10 A. Alamusi, N. Hu, et al., Multi-scale numerical simulations of thermal expansion properties of CNT-reinforced nanocomposites, Nanoscale Res. Lett., 2013, 8, 15.

11 C. D. Brites, et al., Thermometry at the nanoscale, Nanoscale, 2012, 4, 4799-4829.

12 A. Di Bartolomeo, et al., Multiwalled carbon nanotube films as small-sized temperature sensors, J. Appl. Phys., 2009, 105, 064518.

13 Y. Gao and Y. Bando, Carbon nanothermometer containing gallium, Nature, 2002, 415, 599.

14 Y. Gao, Y. Bando and D. Golberg, Melting and expansion behavior of indium in carbon nanotubes, Appl. Phys. Lett., 2002, 81, 4133-4135.

$15 \mathrm{Y}$. $\mathrm{Wu}$ and P. Yang, Germanium/carbon core-sheath nanostructures, Appl. Phys. Lett., 2000, 77, 43-45.

16 F. Arai, et al., in 4th IEEE Conference on Nanotechnology, 2004, pp. 146-148.

17 H. C. Neitzert, L. Vertuccio and A. Sorrentino, Epoxy/MWCNT Composite as Temperature Sensor and Electrical Heating Element, IEEE Trans. Nanotechnol., 2011, 10, 688-693.

$18 \mathrm{~S}$. Hussain, et al., Synthesis of carbon nano-fibers on $\mathrm{p}-\mathrm{Si}$ having improved temperature sensing capability, Mater. Sci. Eng., B, 2013, 178, 83-88.

$19 \mathrm{~K}$. Ali and M. Hafez, Growth and structure of carbon nanotubes based novel catalyst for ultrafast nano-temperature sensor application, Superlattices Microstruct., 2013, 54, 1-6.

20 G. Matzeu, A. Pucci, S. Savi, M. Romanelli and F. D. Francesco, A temperature sensor based on a MWCNT/ SEBS nanocomposite, Sens. Actuators, A, 2012, 178, 94-99.

21 N. J. Blasdel and C. N. Monty, Temperature Sensitive Fabric for Monitoring Dermal Temperature Variations, Wearable electronics sensors: for safe and healthy living, Springer International Publishing, 2015, pp. 193-220.

22 X. Yang, Z. Zhou, F. Zheng and Y. Wu, High sensitivity temperature sensor based on a long, suspended singlewalled carbon nanotube array, Micro \& Nano Letters, 2010, 5, 157-161.

23 S. Selvarasah, et al., A three dimensional multi-walled carbon nanotube based thermal sensor on a flexible parylene substrate, in IEEE Conference on Nanotechnology, Ieee-Nano, 2007, pp. 1062-1066.
24 S. Park, M. Vosguerichian and Z. Bao, A review of fabrication and applications of carbon nanotube film-based flexible electronics, Nanoscale, 2013, 5, 1727.

25 M. Kwon and Y. Hong, Flexible temperature sensor array of PDMS-encapsulated conductive CNT thin films fabricated by solution process, in ISDRS '09. International Semiconductor Device Research Symposium, 2009, pp. 1-2.

26 Y. Zhang, J. Liu and C. Zhu, Novel Gas Ionization Sensors Using Carbon Nanotubes, Sens. Lett., 2010, 8, 219-227.

27 C. Shengbing, Z. Yong and D. Zhemin, Fabrication of gas sensor based on field ionization from SWCNTs with tripolar microelectrode, J. Micromech. Microeng., 2012, 22, 125017.

28 C. Shengbing, D. Zhe min and Z. Yong, Flammable and noxious gas sensing using a microtripolar electrode sensor with diameter and chirality sorted single-walled carbon nanotubes, J. Micromech. Microeng., 2013, 23, 085022.

29 Y. Zhang, et al., High-performance gas sensors with temperature measurement, Sci. Rep., 2013, 3, 1267.

$30 \mathrm{~J}$. Zhang, et al., Properties of a weakly ionized NO gas sensor based on multi-walled carbon nanotubes, Appl. Phys. Lett., 2015, 107, 093104.

$31 \mathrm{X} . \mathrm{Li}$, et al., Carbon nanotube cathode with capping carbon nanosheet, Appl. Surf. Sci., 2013, 283, 740-743.

32 J. Dai, J. Yuan and P. Giannozzi, Gas adsorption on graphene doped with B, N, Al, and S: A theoretical study, Appl. Phys. Lett., 2009, 95, 183.

33 A. S. Ghsemi and F. Ashrafi, Density Functional Theory (DFT) Study of O2, N2 Adsorptions on H-Capped $(4,4)$ SingleWalled Carbon Nanotube, Res. J. Appl. Sci., Eng. Technol., 2012, 4, 2523-2528.

34 S. Jalili and R. Majidi, The effect of atomic hydrogen adsorption on single-walled carbon nanotubes properties, J. Iran. Chem. Soc., 2007, 4, 431-437.

35 J. Zhao, A. Buldum, H. Jie and P. L. Jian, Gas molecule adsorption in carbon nanotubes and nanotube bundles, Nanotechnology, 2001, 13, 3394-3400.

36 M. S. M. Ata, Work function of carbon nanotubes, Carbon, 2001, 39, 1913-1917.

37 C.-W. Chen and M.-H. Lee, Ab initio calculations of dimensional and adsorbate effects on the workfunction of single-walled carbon nanotube, Diamond Relat. Mater., 2003, 12, 565-571.

38 D. Li, et al., First-principles study of H, O, and $\mathrm{N}$ adsorption on metal embedded carbon nanotubes, Appl. Surf. Sci., 2017, 403, 645-651.

$39 \mathrm{H}$. Ago, et al., Work Functions and Surface Functional Groups of Multiwall Carbon Nanotubes, J. Phys. Chem. B, 1999, 103, 8116-8121.

40 S. Suzuki, C. Bower, Y. Watanabe and O. Zhou, Work functions and valence band states of pristine and Csintercalated single-walled carbon nanotube bundles, Appl. Phys. Lett., 2000, 76, 4007-4009. 